\title{
Nuclear Migration and Spindle Formation in the Fourth Cleavage of Sea Urchin Eggs under the Influence of Inhibitors
}

\author{
Gerhard Czihak $^{1}$ and Manabu K. Kojima ${ }^{1,2}$ \\ ${ }^{1}$ Department of Genetics and Developmental Biology, University of Salzburg, Hellbrunnerstr. 34, A-5020 \\ Salzburg, Austria and ${ }^{2}$ Department of Biology, Faculty of Science, Toyama University Toyama 930, Japan
}

Key words: cleavage/sea urchin eggs/asters/mitotic spindle positioning/mitosis inhibitors

\begin{abstract}
$A B S T R A C T$. Mitosis of sea urchin eggs was inhibited when exposed to $3 \mu \mathrm{g} / \mathrm{ml}$ aphidicolin from the 2-cell stage onwards. Nevertheless the nuclei migrated to the vegetal pole at the time of the fourth unequal division in control eggs. Two or four equal or unequal asters developed. Asters in proximity to the vegetal pole were always considerably smaller than those close to the center of the two blastomeres. In contrast to colchicine, cytokinesis but not migration of the nuclei in the vegetal half was prevented by treatments with $5 \mu \mathrm{M}$ cytochalasin B or D. Various mitotic figures were formed in the vegetal blastomeres of eggs treated with $0.4 \mathrm{mM}$ colchicine or $3 \mu \mathrm{M}$ griseofulvin after the third cleavage. In some eggs a centrally localized monaster with chromosomes in spherelike arrangement was formed in others a monopolar mitotic figure pushed the chromosomes in bowl-like arrangements to the most vegetal cortex. In anaphase one set of chromatids migrated to the monopole leaving the scattered sister-chromatids behind. The mechanism of migration of the nuclei and of chromosome arrangement in the metaphase plate is discussed.
\end{abstract}

The fourth cleavage of sea urchin eggs is particular because in early prophase the four nuclei of the vegetal blastomeres "migrate" to the vegetal pole whereas those of the animal blastomeres remain in a central position. Like in radially cleaving eggs of molluscs the eccentric position of the nuclei at the onset of mitosis results in daughter cells of unequal size. K. Dan devoted several studies to aster- and spindle-formation in this unequal cleavage (7-11). T. Schroeder "describes the cytoskeletal machinery during fourth cell division" (28). D. Mazia together with several coworkers published important papers on the formation of monasters and centrosome division $(20,21)$ among which the one of $1986(20)$ is a classic in cell biology. Recently a paper by Holy and Schatten (14) emphasizes the size difference of centrosomes in micromere formation. When the asters in the four vegetal blastomeres are fully developed-that is after the "migration" of the nuclei to the cortex of the vegetal pole, governed by a cortical clock $(7,15)$-we find a big aster at the center of the blastomere and a very reduced and peculiar shaped one close to the cortex of the vegetal pole.

The results presented here clearly show that the "migration" of nuclei is at least in part dependent on microtubules (MTs), that MTs of artificially induced monasters press the chromosomes to the cortex of the vegetal pole and that the perfect planar array of chromosomes in the equatorial plate must be the result of proportionally growing MTs from opposite poles. Our results also confirm the findings of Mazia et al. (21) showing that MTs of a monaster establish contact with only one kinetochore because only one chromatid of each chromosome "migrates" to the monopole.

\section{MATERIALS AND METHODS}

Experiments were performed with the gametes of Paracentrotus lividus from the Northern Adriatics. Basic techniques are described in Czihak (5).

Eggs fixed in ethanol-acetic acid 3:1 at the beginning 16 cell stage were made transparent with $40 \%$ acetic acid for examination with Nomarski interference contrast optics or stained with aceto-carmin or Dapi.

Aphidicolin. This inhibitor or DNA-polymerase $\alpha$ (Sig$\mathrm{ma} / \mathrm{St}$. Louis) was used in concentrations of $2,3,4.5 \mu \mathrm{g} / \mathrm{ml}$ made of a stock solution of $1 \mathrm{mg} / \mathrm{ml}$ dimethylsulfoxide (DMSO).

Colchicine and Griseofulvin. When the majority of eggs had reached the 8 cell stage aliquots were transferred to a 4,2 and $1.25 \times 10^{-4} \mathrm{M}$ solution of colchicine (Merck/Darmstadt, Germany) in ASW and fixed when the controls had reached the 16 cell stage. Griseofulvin (GF) was purchased from Sig$\mathrm{ma}$ (Sigma/St. Louis) and used in a $10 \mathrm{mM}$ stock solution in DMSO and diluted to final concentrations in ASW ranging from 2 to $3.5 \mu \mathrm{M}$.

Cytochalasin B and D. The stock solution of cytochalasin 
Table I. SPINDLE ORIENTATION AND CHROMOSOMAL ARRANGEMENT IN THE VEGETAL 4 BLASTOMERES AT THE 4TH DIVISION IN $3 \mu \mathrm{M}$ GRISEOFULVIN-TREATED EGG.

\begin{tabular}{|c|c|c|c|c|}
\hline \multicolumn{2}{|c|}{ Amphiaster } & \multicolumn{2}{|c|}{ Monaster } & \multirow{3}{*}{$\begin{array}{l}\text { Total number } \\
\text { of observations }\end{array}$} \\
\hline \multicolumn{2}{|c|}{ Spindle orientation } & \multicolumn{2}{|c|}{ chromosomal arrangement } & \\
\hline $\begin{array}{l}\text { perpendicular to } \\
\text { the equator (normal) }\end{array}$ & $\begin{array}{l}\text { at any angle to } \\
\text { the equator }\end{array}$ & Spherical & Bowl shaped & \\
\hline $83(63 \%)$ & $16(12 \%)$ & $14(11 \%)$ & $18(14 \%)$ & $131(100 \%)$ \\
\hline
\end{tabular}

Eggs were put into $3 \mu \mathrm{M}$ Griseofulvin sea water $2 \mathrm{~h} 30 \mathrm{~min}$ after fertilization (when most of them have completed the $3 \mathrm{rd}$ division); $40 \mathrm{mir}$ later, they were fixed with ethanol-acetic acid mixture.

B (CB) and cytochalasin D (CD) (from Sigma/St. Louis) was 0.5 and $1 \mathrm{mM}$ in DMSO. Eggs were transferred into $1.25,2.0$, $2.5,3.75$ and $5 \mu \mathrm{M} \mathrm{CB}$ in sea water $10 \mathrm{~min}$ after completion of the second division and fixed in 10 min intervals after beginning of the 3 rd division in control eggs until control eggs had finished the 4th division.

\section{RESULTS}

Aphidicolin. Brachet and Depetrocellis (3) noted that multipolar mitosis occurred after treatment of fertilized eggs with this inhibitor of DNA-synthesis. We used the drug at the beginning of the 2-cell-stage in order to study the independence of the cycles of DNAsynthesis in the nucleus and the centrosome cycles.

Aphidicolin treated eggs remained at the 2-cell stage. The nuclei increased in size until $3 \mathrm{~h}$ post fertilization. At that time the 4th, unequal cleavage begins in untreated eggs. As in controls the nuclei of aphidicolin treated eggs migrated to one, most likely the vegetal pole. Developing asters were equal or unequal in size

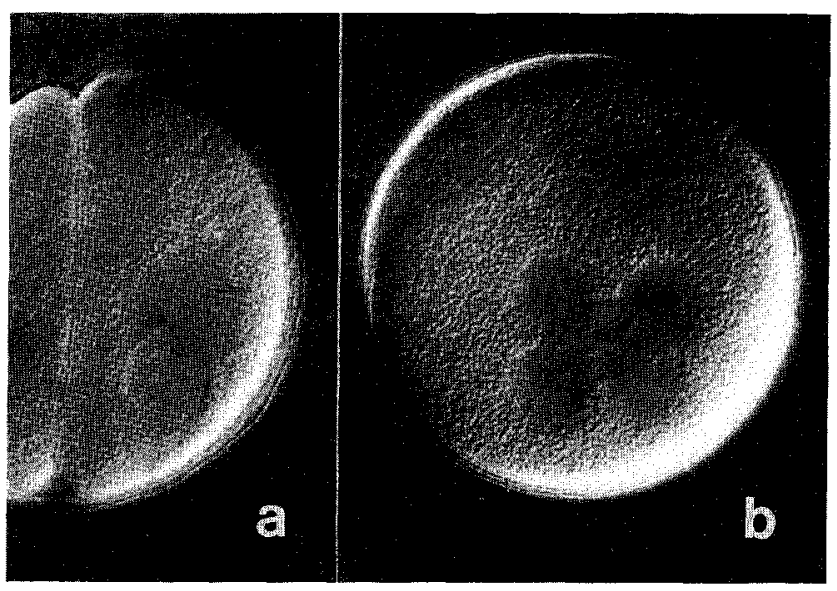

Fig. 1. Mitosis in eggs treated with $2 \mu \mathrm{M}$ aphidicolin since the beginning of the two cell stage at the time of micromere formation in normal eggs. Eggs are still in the 2-cell stage.

a: prophase: the enlarged nucleus migrates to the (most likely) vegetel pole having one large aster in the center and a small one close to the egg's cortex; b: tetrapolar, uniplanar mitosis in the same batch of eggs. $\times 490$
(Fig. 1a, b) As in normally developing eggs those which developed close to the pole were considerably smaller than the one almost in the center of the blastomere (4, $10,21)$. In some eggs tetrapolar cleavage figures appeared at the same time. In this case equally distant asters appear, arranged in one plane and smaller than those in bipolar divisions (Fig. 1b).

In any case the number of centrosomes (CSs) as judged by the number of nucleation sites of asters was reduced in aphidicolin treated eggs. In untreated eggs there is a total of $16 \mathrm{CSs}$ in 8 cells at the beginning of the 4 th cleavage $3 \mathrm{~h}$ post fertilization whereas in aphidicolin treated eggs we found 2 blastomeres with either 2 or 4 asters each, thus 4 or 8 in one egg at the same time.

Effects of cytochalasin $B$ and $D$ on nuclear migration. The migration of the nuclei in the vegetal cells towards the vegetal pole before the 4 th division $(10,15$, 28) was not prevented by these drugs when permanently present since the 4 cell stage and in doses which prevent the formation of a cleavage furrow at the end of the 2 nd division (Fig. 2)

Effects of Colchicine (CO) and Griseofulvin (GF) on Centrosome separation and spindle formation. Fertilized eggs were transferred into $4 \times 10^{-4} \mathrm{M} \mathrm{CO}$ and $3 \mu \mathrm{M}$ GF solutions at the beginning of or when the majority of the eggs had completed the 3rd division.

The results with low doses of $\mathrm{CO}$ and GF were essentially the same.

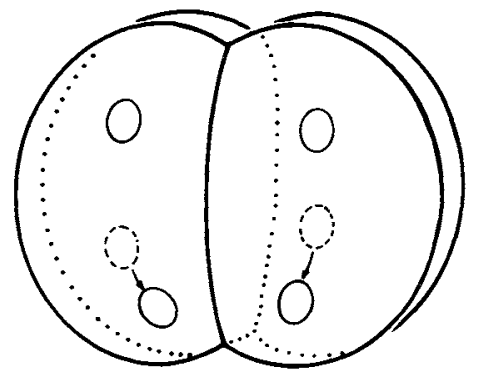

Fig. 2. Prophase of the fourth cleavage in eggs treated with $51 \mathrm{mM}$ cytochalasin B beginning in the 4-cell stage. Cytochalasin B (as does cytochalasin D) prevented the formation of a cleavage furrow after the third mitosis but does not prevent migration of the nuclei to the vegetal pole as indicated by arrows. 


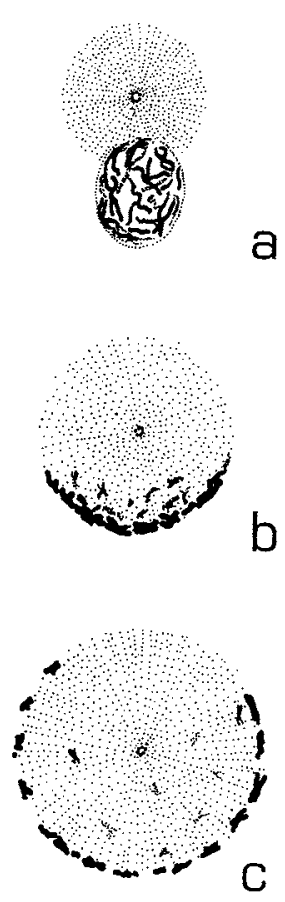

Fig. 3. Three steps $(a-c)$ in the formation of a monaster in the center (!) of a vegetal blastomere in colchicine- or griseofulvin-treated eggs. After disappearance of the nuclear envelope the Centrosome migrates to the center of the previous nucleus and the centrifugally growing microtubules posh the chromosomes towards the periphery: a more or less regular sphere-like arrangement results. In such cases there is no ana-phase movement of chromosomes and the blastomeres remain uncleaved. The situation is different when the monaster is not in the center of the cell (cf. fig. 4).

i. Mitosis in the blastomeres of the animal half appeared normal in as far as amphiaster, spindle formation and anaphase migration of chromosomes were concerned. In some cases, however, spindle orientation was not parallel to the equator as it is in untreated eggs.

ii. In the vegetal half monasters or amphiasters were formed. In most cases they were of different types and sizes in the same egg and exhibited the following characteristics:

iia. The migration of the nuclei to the vegetal pole often was found blocked in only one or two blastomeres of the same egg if at all. Nuclei that remained in the center of a vegetal blastomere could revert to a cleavage figure with an amphiaster composed of equally sized asters (!) and with a spindle oriented at any angle to the equator (approx. 12\%, Table I). In other cases (approx. 11\%, Table I) a centrally localized monaster was formed in which the chromosomes were arranged like on a surface of a sphere (Fig. 3).

iib. Nuclei which migrated to the most vegetal part of the blastomere participated in the formation of either a normally oriented division figure (approx. $63 \%$, Table I) with a very small aster at the vegetal pole and a

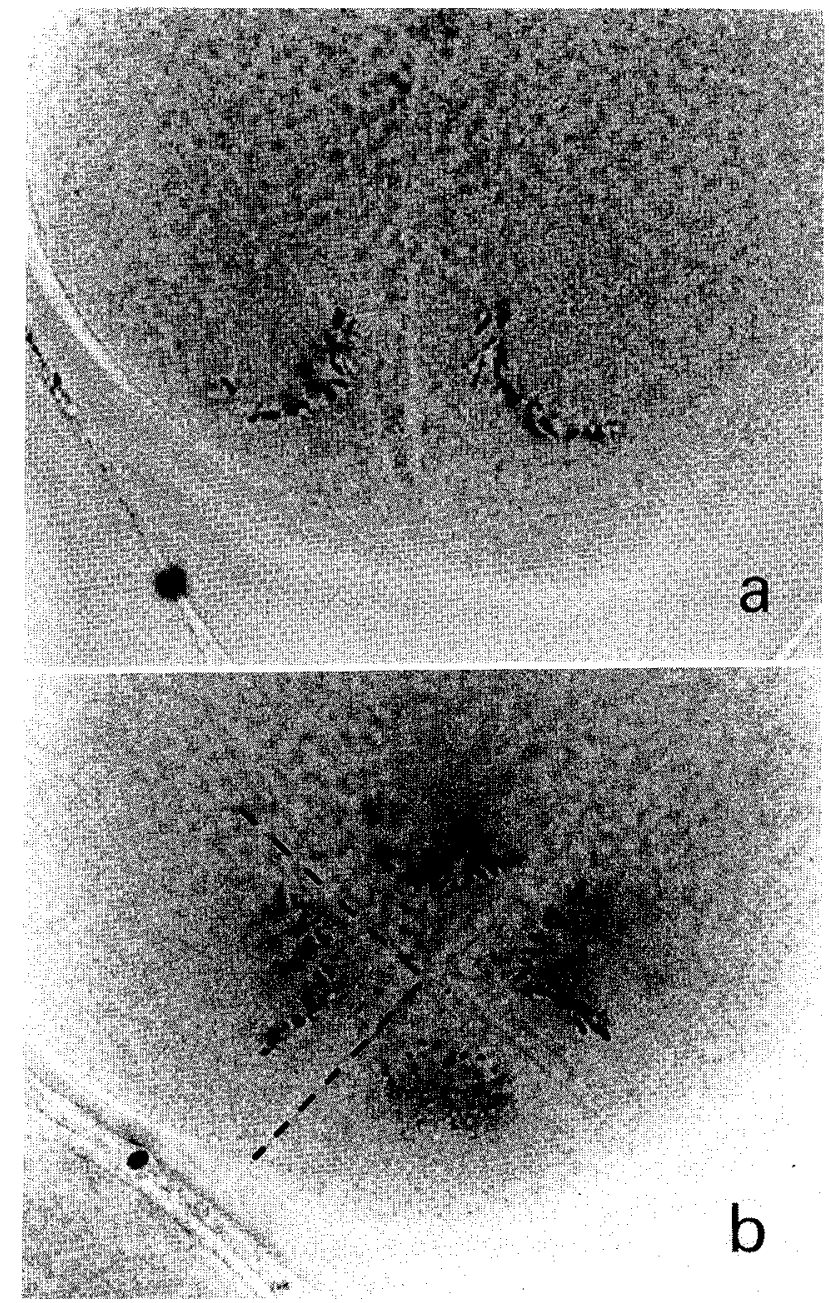

Fig. 4. Metaphase of monopolar mitotic figures in vegetal blastomeres treated with $4 \times 10^{-4} \mathrm{M}$ colchicine at the time of micromere formation; aceto-carmine staining. Chromosomes are pushed to the vegetal pole (a) and finally pressed to the vegetal cortex (b). Cell boundaries in one blastomere are emphazized with black dashes to show the thickness of the cortical cytoplasm into which the chromosomes do not enter. $\times 1000$

large one at the opposite pole in the center of the vegetal blastomere or a monaster was formed (approx. 14\%, Table I) with chromosomes arranged in the form of a bowl. Later the chromosomes were found pushed to the most vegetal cortex (Fig. 4). In anaphase one set of chromatids migrated towards the spindle pole leaving the sister chromatids behind. Half spindles were often barrelshaped, thus very much broadened (Fig. 5 and 6). Two groups of nuclear vesicles formed, one at the broadened spindle pole, the other seemingly "attached" to the vegetal cortex. Subsequently the two clusters of nuclear vesicles fused to two nuclei. There was no cytokinesis and the vegetal blastomeres were therefore binucleate. 


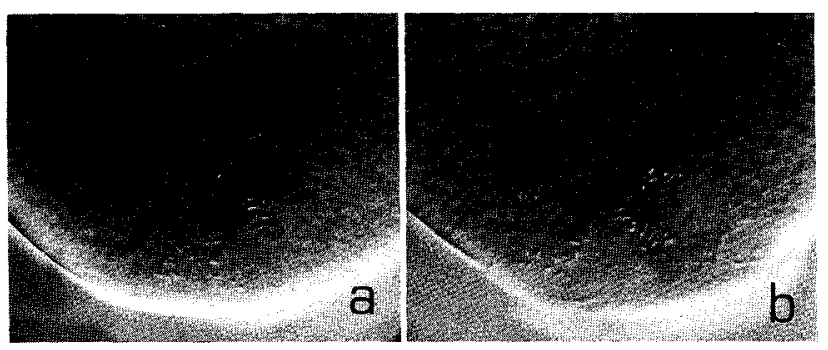

Fig. 5. Anaphase in griseofulvin treated eggs $(3 \mu \mathrm{M})$ in two focal planes $(a, b)$ of the same cells seen in Nomarski interference contrast. The upper set of chromatids (clearly visible in the blastomere at the left) "migrating" in linear arrangement towards the pole of the monopolar spindle. The sister chromatids remain scattered at the vegetal cortex. Note the broad front of "migrating" chromatids. $\times 560$

\section{DISCUSSION}

The independence of the centrosome and chromosome cycles in sea urchin eggs as confirmed by our aphidicolin experiments was already shown by Rattner and Phillips (25), Mazia et al. (21), Czihak et al. (6) and others. We have no explanation of the fact that sometimes two, sometimes four asters developed under identical experimental conditions (Fig. 1). Asters contain centrosomal MTOCs. In such cases the position of asters follow the rule of equal distance (6). Four asters with centrosomes stemming from one spermatozoon were arranged in one circle (Fig. 1b) in contrast to dispermic eggs in which the asters are frequently positioned like in the corners of a tetrahedron.

In all experiments which prevent migration of the nucleus to the vegetal pole $3 \mathrm{~h}$ after fertilization in Paracentrotus lividus eggs at $20^{\circ} \mathrm{C}$ asters of equal size are formed in the fourth cleavage. If, however, nuclei do "migrate" to the periphery of the vegetal blastomerethis canbe in a delayed 2-, 4- or 8-cell stage-the astral rays coming close to the vegetal pole are much reduced in size $(4,9,21)$. In experiments with Hemicentrotus pulcherrimus $(17,18)$ aphidicolin also prevented mitosis: eggs remained at the 1-cell stage when controls had com-

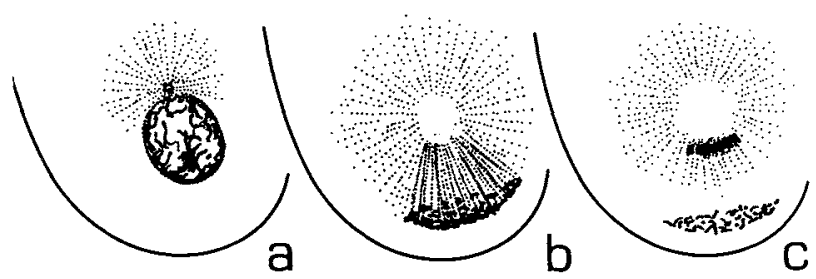

Fig. 6. Schematic drawings to make events shown in fig. 5 comprehensible.

a: prophase (not shown in a photograph); b: metaphase (cf. fig. 4); c: anaphase (cf. fig. 5).
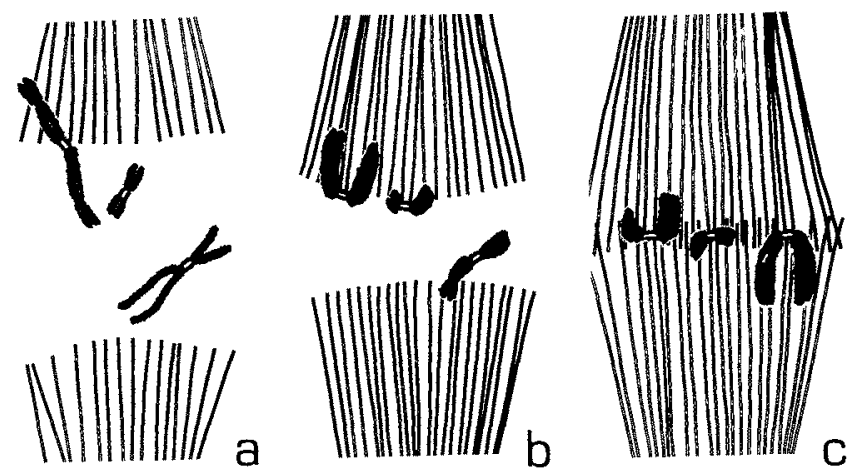

Fig. 7. Schematic drawing of several steps in the transition from prometa- to metaphase to illustrate the hypothesis of chromosome arrangement in the metaphase plate. Scattered chromosomes are pushed towards the equatorial plane when growing spindle fibers established contact to one kinetochore of a chromosome somewhere between the poles. This movement comes to a standstill when the partner-kinetochore adhere to spindle fibers from the opposite pole. MTs without contact to chromosomes can grow further, overlapping each other or even reaching the opposite pole, most likely until the tubulin pool is exhausted. Only three different chromosomes are shown.

a. the metacentric chromosome 1 establishes contact with a microtubule by one of its kinetochores; $b$. chromosome 1 and 3 in unilateral contact with MTs and shifted towards the equator; c. chromosomes 1-3 in contact with MTs from both poles of the bipolar mitotic figure in the equatorial plane $=$ metaphase plate, chromosome translocation stopped, tips of MTs without contact to kinetochores growing further and overlapping.

pleted 3 cell cycles.

This is in contrast to experiments with the eggs of the starfish Asterina pectinifera: In presence of aphidicolin chromosomes condense at the time of first cleavage, become arranged in a metaphase plate but do not " $\mathrm{mi}$ grate" to the normally developed poles. Nevertheless cytokinesis can be completed and chromatin clumps remain at the cleavage furrow $(24,27,31)$. This striking difference in nuclear behavior under the influence of aphidicolin is certainly worthwile to be explored further.

When respect to our experiments, two conclusions can be drawn:

i. There must be an internal clock $(7,14)$ which controls the ventralwards translocation of the nuclei. The area where micromeres are formed cannot be shifted by centrifugation (23). The clock is therefore thought to reside in the plasma membrane or the rather stiff cortex (traditionally called cortical clock). In the fifth cleavage even smaller micromeres are formed. The clock is apparently turned on between 3 and $4 \mathrm{~h}$ after fertilization independent of the number of blastomeres already formed.

ii. Size of asters seem to depend on availability of tubulin subunits. Asters become smaller with decreasing size of blastomeres throughout cleavage as well as in ar- 
tificially formed minicells by making exovates mechanically (4).

Asters of one cleavage figure are of different size when the MTs of the most vegetal aster reach the vegetal cortex suggesting that they become disaggregated at the vegetal cortex or stopped in growth. Asters can be of equal size when the cleavage figure is positioned close to the center and parallel to the equator in a vegetal blastomere of the same egg age. More details on aster formation in the fourth cleavage are given by Schroeder (28) and Holy and Schatten (14).

Our results with cytochalasins confirm the findings of Tanaka (29) who showed that neither migration of nuclei nor micromere formation can be prevented when eggs are exposed to cytochalasin B at the 2- or 4-cell stage.

When a monaster forms under the influence of colchicine or GF in the center of a vegetal blastomere approximately $3 \mathrm{~h}$ p.f. the chromosomes become arranged in form of an incomplete sphete (Fig. 3). Such monasters persist for hours and such blastomeres remain uncleaved. This is in accordance with results of Mazia et al. (21) and Wang et al. (30). Chromosomes "do split apart ... do not separate further" (p. 379). "Chromosomes are distributed in a spherical shell ... without chromatid separation" (p. 301).

Ito and Sato (19), however, reported recently that chromosomes in half spindles induced by $\beta$-mercaptoethanol do move towards the monopole.

Monasters at the vegetal pole demonstrated that chromosomes are captured by MTs $(12,22)$ and thrusted $(1$, 2) to the vegetal cortex.

This observation allows the conclusion that the normally perfect arrangement of chromosomes in the equatorial plane at metaphase is brought about by two equally developing bundles of spindle fibers coming from opposite poles. The passive "migration" of chromosomes comes to a standstill when the growth of microtubules attached to the kinetochores is annihilated by equal forces from both sides (Fig. 7). This hypothesis needs an additional assumption: the force of a MT shifting a chromosome should be lesser the more the MT elongates. In such a way chromosomes that were pushed somewhat over the equatorial plane could be brought back to it.

In mercaptoethanol-, colchicine- and GF-monasters only one chromatid of a pair can establish contacts to MTs of the spindle. For mercaptoethanol it was shown electromicroscopically (21); we conclude it from the fact that there is only one halfspindle and consequently under the influence of GF only one set of chromatids migrates to the monopole leaving the other set of chromosomes widely distributed at the vegetal cortex. Anaphase movement of chromosomes was not found in colchicine induced monasters.
At the moment we cannot explain, why in eccentric positioned monasters sister chromatides do migrate whereas in centrally positioned monasters sister chromatides do not.

In many colchichine- and GF- treated blastomeresnot only in the vegetal ones-the spindle poles are found extremely broadened and the spindle axis shortened (Figs. 5, 6), resulting in barrelshaped cleavage figures.

The above mentioned, but not verified hypothesis according to which microtubules become depolymerized at the vegetal pole allowing the MTs of the central aster to grow disproportionally and pushing the nucleus towards the vegetal pole (4) was put into question by Schroeder (28), because asters at the end of prophase "... are not very extensive, so it is unlikely .. that migration is accomplished merely by ... differential growth." But there can be no doubt that normal asters in thte proximity of the vegetal pole appear weakened and are distinctly reduced in size and disappear when microtubules disintegrate under the influence of colchicine or GF in concentrations in which they do not influence stable microtubules in the inner part of the vegetal or in the animal blastomeres. Future experiments with not yet available antibodies against sea urchin centrosomes can help to answer the question whether centrosomes duplicate and migrate to opposite poles in experimentally induced monasters.

A plausible explanation for the results with colchicine and griseofulvin, administered before the fourth cleavage is, that one centrosome becomes perfectly well positioned between the eccentric nucleus and the most vegetal cortex and furthermore that a half spindle nucleated by this centrosome is a very rudimentary one which rapidly disintegrates under the influence of the cortex and the drugs. Therefore only the halfspindle more distant from the cortex persists allowing anaphase-movement of one set of chromatides.

In convincing experiments of Hiramoto and Shoji (13) and Nicklas (26) it was shown that a persistant mitotic pole is not a prerequisite for anaphase movement of chromosomes.

Acknowledgements. First of all we thank the reviewers for their excellent comments and Mrs. E. Fimberger for her support in all laboratory techniques and Mrs. $\mathrm{H}$. Oder for the maintenance of the sea urchin flocks keeping the spines upright for many months. L. Slomkowsky made part of the photographs.

\section{REFERENCES}

1. BaJer, A.S. (1982). Functional autonomy of monopolar spindle and evidence for oscillatory movement in mitosis. $J$. Cell Biol., 95: 33-48.

2. Bajer, A.S., Cypher, C., Mole-Bajer, J., and Howard, H.M. (1982). Taxol-induced anaphase reversal: eveidence that elon- 
gating microtubules can exert a pushing force in living cells. Proc. Natl. Acad. Sci. USA, 79: 6569-6573.

3. Brachet, J. and DePetrocellis, B. (1981). The effects of aphidicolin, an inhibitor of DNA replication, on sea urchin development. Exp. Cell Res., 135: 179-189.

4. CzinaK, G. (1973). The role of astral rays in early cleavage of sea urchin eggs. Exp. Cell Res., 83: 424-426.

5. CzIHAK, G. (edit.) (1975). The sea urchin embryo. Biochemistry and morphogenesis. 700p Heidelberg.

6. CzihaK, G., Kojima, M., Linhart, J., and Vogel, H. (1991). Multipolar mitosis in procaine-treated polyspermic sea urchin eggs and in eggs fertilized with UV-irradiated spermatozoa with a computer model to simulate the positioning of centrosomes. Europ. J. Cell Biol., 55: 255-261.

7. DAN, K. (1971). On the system controlling the time of micromere formation in sea urchin embryos. Developm. Growth Different., 13: 285-301.

8. DAN, K. (1972). Modified cleavage pattern after supression of one mitotic division. Exp. Cell Res., 72: 69-73.

9. DAN, K. (1979). Studies on unequal cleavage in sea urchins. I. Migration of the nuclei to the vegetal pole. Developm. Growth Different., 21: 527-535.

10. DAN, K. (1984). The cause and consequence of unequal cleavage in sea urchins. Zool. Sci., 1: 151-160.

11. Dan, K., Endo, S., and Uemura, I. (1983). Studies on unequal cleavage in sea urchins. II. Surface differentiation and the direction of nuclear migration. Developm. Growth Different., 25: $227-237$.

12. HAYDEN, J.H., Bowser, S.S., and RIEDER, C.L. (1990). Kinetochores capture astral microtubules during chromosome attachment to the mitotic spindle: direct visualization in live newt lung cells. J. Cell Biol., 111: 1039-1045.

13. HiRAmoto, Y., and Shoj, Y. (1982). Location of the motive force for chromosome movement in sand dollar eggs. Cell Differentiation, 11: 349-351.

14. Holy, J. and Schatten, G. (1991). Differential behavior of centrosomes in unequally dividing blastomeres during fourth cleavage of sea urchin embryos. $J$. Cell Sci., 98: 423-431.

15. Horstadius, S. (1973). Experimental embryologyt of echinoderms. 192p. Oxford.

16. Hyman, A.A., and Mrtchison, T.J. (1991). Two different microtubule-based motor activities with opposite polarities in kinetochores. Nature, 351: 206-209.

17. Ikegami, S., Amemiya, S., Oguro, M., Nagano, H. and Mano, Y. (1979). Inhibition of cell cycle progression and DNS replication in sea urchin embryos. J. Cell Physiol., 100: 439-444.
18. Ikegami, S., Taguchi, T., Ohashi, M., Oguro, M., Nagano, H. and MANO, Y. (1978). Aphidicolin prevents mitotic cell division by interfering with the activity of DNA polymerase- $\alpha$. $N a$ ture, 275: $458-460$.

19. Iто, K. and SAто, H. (1991). Anaphase chromosome movement in the sea urchin monopolar spindle. Cell Struct. Funct., 16: 554

20. MAZIA, D. (1986). The chromosome cycle and the centrosome cycle in the mitotic cycle. Int. Rev. Cytol., 100: 49-92.

21. Mazia, D., Paweletz, N., Sluder, G. and Finze, E.-M. (1981). Cooperation of kinetochores and pole in the establishment of monopolar mitotic apparatus. Proc. Natl. Acad. Sci. USA, 78: $377-381$.

22. Mrtchison, T.J. (1990). The kinetochore in captivity. Nature, 348: $14-15$

23. Morgan, T.H. and Spooner, G.B. (1909). The polarity of the centrifuged egg. W. Roux's Arch. Entwicklungsmech. Organismen 28: 104-117.

24. Nagano, H., Hirai, S., Okano, K. and Ikegami, S. (1981). Achromosomal cleavage of fertilized starfish eggs in the presence of aphidicolin. Dev. Biol., 85: 409-415.

25. RattNer, J.B. and Philips, S.G. (1973). Independence of centriole formation and DNA-synthesis. J. Cell Biol., 57: 359-372.

26. NICKLAS, R.B. (1989). The motor for poleward chromosome movement in anaphase is in or near the kinetochore. $J$. Cell Biol., 109: 2245-2255.

27. Saiki, T., KYozuka, K., Osanai, K., Hamaguchi, Y. (1991). Chromosomal behavior in starfish (Asterina pectinifera) zygotes under the effect of aphidicolin, an inhibitor of DNA polymerase. Exp. Cell Res., 192: 380-388.

28. SCHROEDER, T. (1987). Fourth cleavage of sea urchin blastomeres: Microtubule patterns and myosin localization in equal and unequal cell divisions. Dev. Biol, 124: 9-22.

29. TANAKA, Y. (1981). Distribution and redistribution of pigment granules in the development of sea urchin embryos. Roux's Arch. Develop. Biol., 190: 267-273.

30. WANG, R.J., Wissinger, W., King, E.J. and WANG, G. (1983). Studies on cell division in mammalian cells. VII. A temperature-sensitive cell line abnormal in centriole separation and chromosome movement. J. Cell Biol., 96: 301-306.

31. Yamada, H., Hirai, S., IKegami, S., Kawarda, Y., Okuhara, E. and NAGANO, H. (1985). The fate of DNA originally existing in the zygote nucleus during achromosomal cleavage of fertilized echinoderm eggs in the presence of aphidicolin: microscopic studies with anti-DNA antibody. J. Cell Physiol., 124: 9-12.

(Received for publication, June 16, 1991

and in revised form, March 16, 1992) 